\title{
REPLAYING THE 2001 OUTBURST OF WZ SGE
}

\author{
D.Steeghs ${ }^{1}$ \\ RESUMEN
}

Presento los resultados clave de la espectroscopía óptica extensa obtenida durante las primeras tres semanas de la superexplosión en 2001 de WZ Sge. Se construyó una secuencia de mapas Doppler del flujo de acreción en varias líneas, y se utilizó para crear películas en tiempo real de WZ Sge conforme avanzaba su evolución a través de la explosión. Los principales resultados incluyen la detección de asimetrías espirales de dos brazos en el disco, emisión irradiada desde la estrella donadora y una extensa corriente de emisión alrededor del día 19 después de la explosión que muestra huellas de un evento explosivo con transferencia de masa.

\begin{abstract}
I present the key results of extensive optical spectroscopy obtained during the first three weeks of the 2001 super-outburst of WZ Sge. A sequence of Doppler maps of the accretion flow in various lines was constructed, and used to create real-time movies of WZ Sge as it evolved through the outburst. Main results include the detection of two-armed spiral asymmetries in the disk, emission from the irradiated donor star and an extended stream of emission around day 19 of the outburst that bears the hallmarks of a mass-transfer burst event.
\end{abstract}

Key Words: ACCRETION, ACCRETION DISKS - NOVAE, CATACLYSMIC VARIABLES — STARS: INDIVIDUAL (WZ SGE)

On July 23rd 2001, the short period dwarf nova WZ Sge went into one of its large-amplitude outbursts, surprising us all with its premature activity. An intensive observing campaign was launched at many wavelengths in order to capture as much of this rare event. I describe time-lapsed Doppler tomography results obtained from extensive optical spectroscopy of WZ Sge during the first three weeks of its outburst.

The spectroscopy described here was obtained using the INT and WHT telescopes on La Palma, with their IDS and ISIS spectrographs respectively. Target spectra were normalized using a spline-fit to the continuum after optimal extraction. We constructed phase-binned line profiles by applying the Patterson et al. (1998) ephemeris and folding the spectra into 125 bins per 82 minute orbit. Doppler tomograms were calculated whenever suitable phaseresolved data was at hand, amounting to 11 nights covering the first 21 days of the outburst. This unique data set thus gave us the opportunity to construct real-time movies of the changing accretion flow dynamics in WZ Sge during its outburst. Figure 1 displays several representative Doppler maps that were generated from the data. Doppler tomography is ideally suited for structures that are fixed in the co-rotating frame of the binary (Marsh 2001; Steeghs 2003). However, in super-humping systems

\footnotetext{
${ }^{1}$ Harvard-Smithsonian Center for Astrophysics
}

such as WZ Sge, which started to display superhumps around day 13 of the outburst, the disk is thought to precess in the binary frame when superhumps are observed. Mapping for example the super-hump light from such a precessing disk is not something that standard Doppler tomography is directly suited for, and I thus focus here on the corotating emission structures in WZ Sge.

Movies of the changing Doppler maps of the HeII $4686 \AA$ emission line as well as $\mathrm{H} \beta$ were created by linearly interpolating the maps in time to generate a smooth movie with a constant frame-rate ${ }^{2}$. I attempt here to briefly describe the key features of these movies. The ionized helium line is a prominent spectral feature early during the outburst (see also Nogami \& Iijima 2004). The Doppler maps indicate it originates in a highly asymmetric accretion disk. The distorted disk with its two-armed emission pattern closely resembles the spiral structures that have been observed in other dwarf novae in outburst, and are thought to be driven by the tides of the mass donor star on the large and hot accretion disk (e.g. Steeghs 2001). The Balmer lines consist of both emission as well as absorption components that can cut into the extended disk emission, and show a similar asymmetric disk contribution. This double-armed structure in the maps is accompanied

\footnotetext{
${ }^{2}$ The full movies themselves are available on-line at http://hea-www. cfa. harvard. edu/ dsteeghs/wzsge/movies.html
} 
by a double-humped orbital photometric lightcurve during this early stage of the outburst (Patterson et al. 2002), suggesting a common cause (see also Osaki \& Meyer 2002). HeII emission persists until $\sim 19$ days into the outburst, when the line flux drops to zero, indicative of a cooled down accretion disk compared to its outburst-maximum state. This gradual decrease in the HeII flux is accompanied by the disappearance of the two-armed spirals, except for a short lived re-brightening that occurs around day 14, during which the line emission from the disk temporarily increases and the two-armed spiral structure returns for a day or two before fading away completely. Although the overall evolution of the disk emission in the Balmer lines is very similar to that of HeII, a key additional source is present. This can be attributed to emission from the irradiated front of the mass donor star, providing us with the first view of this faint low-mass object (Steeghs et al. 2001). The strength of the donor star emission is variable, and after fading in strength initially, it recovers two weeks into the outburst. This recovery is followed by a spectacular change in the Doppler maps around day 18, when an extensive stream of emission appears bridging the donor star and the outer disk. When comparing the kinematics of this stream with the expected velocities of the ballistic stream, the agreement is remarkable (Fig.1; lower right panel), especially since the trajectory of the ballistic stream is fixed independently by the emission from the mass donor star. Also, the strength and shape of the feature evolves strictly along the stream trajectory. A natural explanation for this emission could thus be a burst of mass from the heated donor star, producing the extra stream emission as its interacts with the disk. Such a burst has been proposed in the past to explain the peculiar outburst properties of WZ Sge, and it is furthermore supported by the coincident detection of a strong 'bright-spot' eclipse in optical photometry that points to a 60 fold increase in the bright-spot emission (Patterson et al. 2002). Alternatively, the additional stream emission may be produced due to an enhanced interaction with the outer disk (see also Osaki \& Meyer 2003). However, this interaction is expected to severely distort the kinematics of the stream away from its ballistic velocities, but no such departure is observed. Also, the diskstream interaction due to the precessing disk does indeed modulate the emission as a function of the super-hump cycle, but this modulation is of a much smaller amplitude and thus one would need to postulate that this interaction is somehow anomalously strong only during this short-lived phase despite the
Hell 4686 $+\beta$

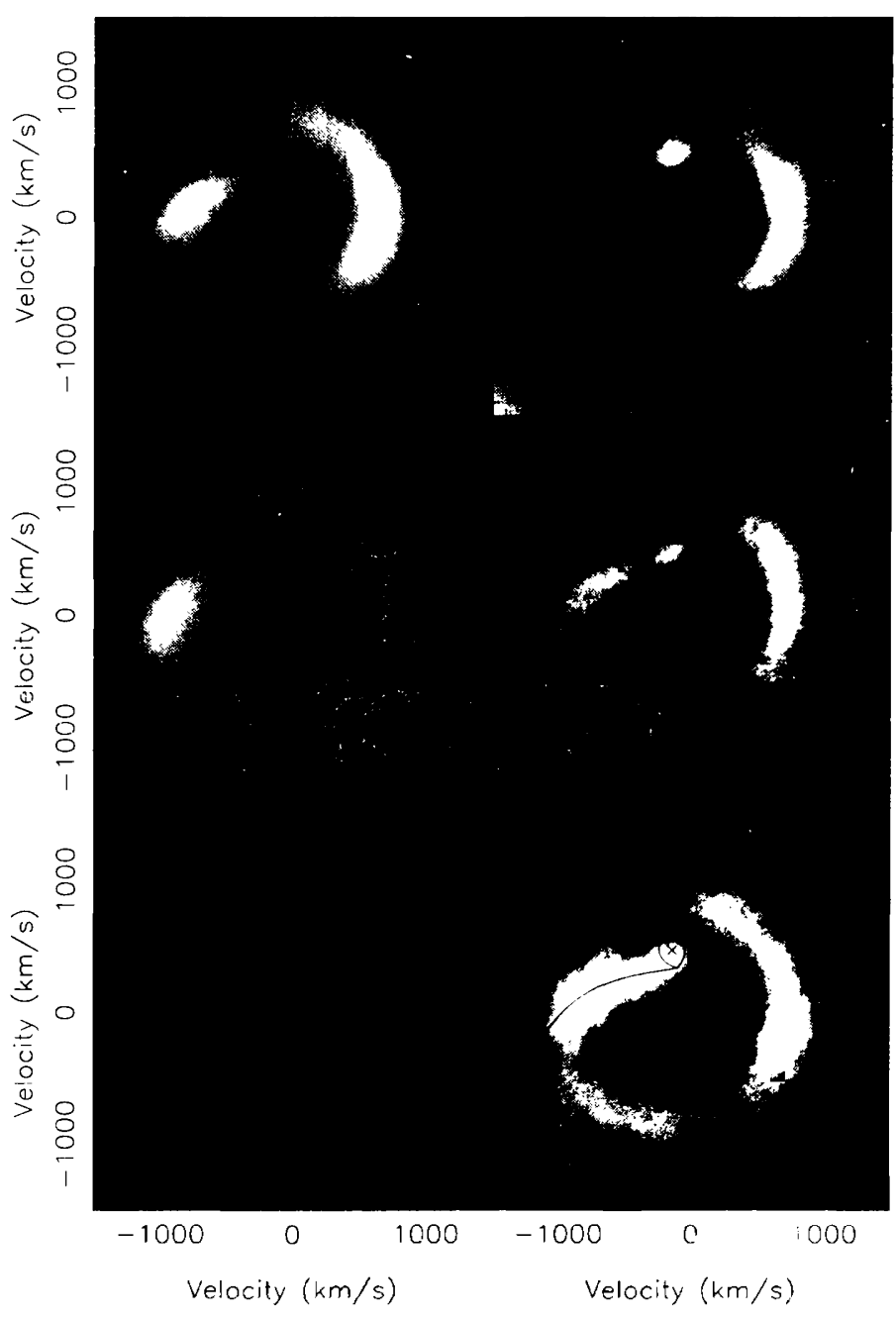

Fig. 1. Representative Doppler maps of WZ Sge in the light of Hell $4686 \AA$ (left) and $\mathrm{H} \beta$ on days 3 (top), 9 (middle) and 19 of the outburst.

fact that super-humps are present for months. These first-generation Doppler movies illustrate the potential of time-lapsed tomography experiments. A more extensive analysis of this data together with spectroscopy obtained at other telescopes is in preparation.

DS acknowledges a SAO Clay Fellowship.

\section{REFERENCES}

Marsh, T.R, 2001, in: Boffin, Steeghs \& Cuypers, Astrotomography, LNP Series 573, Springer Verlag. Berlin Nogami, D., Iijima, T., 2004, PASJ, in press Osaki, Y., Meyer, F., 2003, A\&A, 401, 32: Osaki, Y., Meyer, F., 2002, A\&A, 383, 57t Patterson, J. et al., 1998, PASP, 110, 403 Patterson, J. et al., 2002, PASP, 11.4. 721

Steeghs, D., 2003, MNRAS, 344, 448

Steeghs, D. et al.. 2001. ApJ, 562. 145

Steeghs, D., 2001, in: Boffin. Steeghs of Cuypers, Astrutomography, LNP Series 573, Springer Verlag. Berlin 